Supporting information for:

\title{
Virtual Screening against Metalloenzymes for Inhibitors and Substrates ${ }^{\S}$
}

\author{
John J. Irwin ${ }^{+}$, Frank M. Raushel ${ }^{\&}$ and Brian K. Shoichet ${ }^{+*}$
}


Table S1: Docking statistics

\begin{tabular}{|c|c|c|c|c|c|}
\hline Enzyme & $\begin{array}{c}\text { Unique } \\
\text { Molecules } \\
\text { Scored }\end{array}$ & $\begin{array}{c}\text { Total } \\
\text { Molecules } \\
\text { Scored* }\end{array}$ & $\begin{array}{l}\text { Average } \\
\text { Orientations } \\
\text { Sampled per } \\
\text { molecule }\end{array}$ & $\begin{array}{c}\text { Total } \\
\text { configurations } \\
\text { scored }\left({ }^{\circ} 000\right)^{\mathrm{a}}\end{array}$ & $\begin{array}{c}\text { Total } \\
\text { time }(h)^{b}\end{array}$ \\
\hline CA II & 87963 & 116251 & 4313 & 280065 & 89.5 \\
\hline MMP-3 & 91218 & 127953 & 5017 & 205189 & 51.1 \\
\hline NEP & 46616 & 65411 & 6482 & 464629 & 82.9 \\
\hline PDF & 92418 & 128485 & 3924 & 317404 & 84.3 \\
\hline XO & 51104 & 64923 & 5928 & 303629 & 5.6 \\
\hline $\mathrm{ZnBL}^{\mathrm{c} 1}$ & 33812 & 33812 & 11206 & 1102738 & 127.5 \\
\hline $\mathrm{PTE}^{\mathrm{c} 2}$ & 79393 & 134321 & 3838 & 2921030 & 225.9 \\
\hline
\end{tabular}

a. Only orientations and conformations passing the steric filter were scored.

b. Scaled to reflect time on a single Intel $2.4 \mathrm{GHz}$ Xeon processor

c. 1. Docked to ZINC fragment-like subset. 2. Docked to ACD. All others docked to MDDR.

* includes alternate protonation states and tautomers 
Table S2. Comparison of atomic charge parameters for phosphotriesterase (1hzy).

\begin{tabular}{|c|c|c|c|c|c|}
\hline $\begin{array}{l}\text { Residue } \\
\text { Identity }\end{array}$ & $\begin{array}{l}\text { PDB Atom } \\
\text { Name }\end{array}$ & $\begin{array}{l}\text { AMBER united } \\
\text { atom charge }\end{array}$ & $\begin{array}{l}\text { Atom labels } \\
\text { in the Jaguar } \\
\text { input }\end{array}$ & $\begin{array}{l}\text { Jaguar-calculated } \\
\text { atomic charges }\end{array}$ & $\begin{array}{l}\text { Adapted } \\
\text { AMBER } \\
\text { charges by } \\
\text { our method }\end{array}$ \\
\hline \multirow[t]{5}{*}{ His55 } & CG & 0.089 & $\mathrm{C} 1+\mathrm{H} 6$ & $-0.102+0.441$ & 0.089 \\
\hline & ND1+HND & $-0.444+0.320$ & $\mathrm{~N} 2+\mathrm{H} 7$ & $-0.395+0.162$ & -0.120 \\
\hline & CD2 & 0.145 & $\mathrm{C} 3+\mathrm{H} 8$ & $-0.061+0.101$ & 0.145 \\
\hline & CE1 & 0.384 & $\mathrm{C} 4+\mathrm{H} 9$ & $0.369+0.223$ & 0.384 \\
\hline & NE2 & -0.527 & $\mathrm{~N} 5$ & -0.586 & -0.327 \\
\hline \multirow[t]{5}{*}{ His57 } & CG & 0.089 & $\mathrm{C} 10+\mathrm{H} 15$ & $-0.248+0.390$ & 0.089 \\
\hline & ND1+HND & $-0.444+0.320$ & $\mathrm{~N} 11+\mathrm{H} 16$ & $-0.223+0.136$ & -0.120 \\
\hline & CD2 & 0.145 & $\mathrm{C} 12+\mathrm{H} 17$ & $0.027+0.150$ & 0.145 \\
\hline & CE1 & 0.384 & $\mathrm{C} 13+\mathrm{H} 18$ & $0.159+0.220$ & 0.384 \\
\hline & NE2 & -0.527 & N14 & -0.419 & -0.327 \\
\hline \multirow[t]{2}{*}{ Lys169 } & $\mathrm{CE}$ & 0.218 & $\begin{array}{l}\mathrm{C} 19+\mathrm{H} 21 \\
+\mathrm{H} 22+\mathrm{H} 23 \\
\end{array}$ & $\begin{array}{l}0.090+0.111+ \\
0.081+0.132\end{array}$ & 0.218 \\
\hline & NZ & -0.272 & $\mathrm{~N} 20+\mathrm{H} 51$ & $-0.808+0.434$ & -0.272 \\
\hline \multirow[t]{3}{*}{ CBX } & $\mathrm{C}$ & 0.0 & $\mathrm{C} 24$ & 1.158 & 0.0 \\
\hline & O1 & -0.5 & $\mathrm{O} 25$ & -0.861 & -0.7 \\
\hline & $\mathrm{O} 2$ & -0.5 & $\mathrm{O} 26$ & -0.895 & -0.7 \\
\hline \multirow[t]{5}{*}{ His201 } & CG & 0.089 & $\mathrm{C} 27+\mathrm{H} 32$ & $0.068+0.374$ & 0.089 \\
\hline & ND1+HND & $-0.444+0.320$ & $\mathrm{~N} 28+\mathrm{H} 33$ & $-0.741+0.204$ & -0.120 \\
\hline & CD2 & 0.145 & $\mathrm{C} 29+\mathrm{H} 34$ & $-0.149+0.162$ & 0.145 \\
\hline & CE1 & 0.384 & $\mathrm{C} 30+\mathrm{H} 35$ & $0.282+0.149$ & 0.384 \\
\hline & NE2 & -0.527 & N31 & -0.222 & -0.327 \\
\hline \multirow[t]{5}{*}{ His230 } & CG & 0.089 & $\mathrm{C} 36+\mathrm{H} 41$ & $-0.048+0.445$ & 0.089 \\
\hline & ND1+HND & $-0.444+0.320$ & $\mathrm{~N} 37+\mathrm{H} 42$ & $-0.463+0.132$ & -0.120 \\
\hline & CD2 & 0.145 & $\mathrm{C} 38+\mathrm{H} 43$ & $-0.074+0.107$ & 0.145 \\
\hline & CE1 & 0.384 & $\mathrm{C} 39+\mathrm{H} 44$ & $0.395+0.203$ & 0.384 \\
\hline & NE2 & -0.527 & $\mathrm{~N} 40$ & -0.542 & -0.327 \\
\hline \multirow[t]{3}{*}{ Asp301 } & CG & 0.620 & $\mathrm{C} 45+\mathrm{H} 48$ & $0.914+0.029$ & 0.620 \\
\hline & OD1 & -0.706 & $\mathrm{O} 46$ & -0.801 & -0.906 \\
\hline & OD2 & -0.706 & O47 & -0.948 & -0.906 \\
\hline Wat & $\mathrm{O}$ & -1.0 & $\mathrm{O} 50+\mathrm{H} 49$ & $-1.343+0.548$ & -1.0 \\
\hline $\mathrm{Zn} 1$ & $\mathrm{ZN}$ & 2.0 & ZN1 & 1.221 & 1.4 \\
\hline $\mathrm{Zn} 2$ & $\mathrm{ZN}$ & 2.0 & $\mathrm{ZN} 2$ & 1.471 & 1.4 \\
\hline
\end{tabular}

Notes on Table S2: We prepared a truncated model of the binding site of phosphotriesterase (PDB 1hzy) as in the PDB file below. We performed an ab initio molecular orbital calculation at the Hartree-Fock level with the LACVP basis set in the Jaguar program (Schrodinger, Inc). The input is included below. This table compares the AMBER united atom charge model, the charge distribution from Jaguar calculation, and 
our simplistic adaptation of the Amber charge model as describe in the main body of the paper. The experience reported in this paper supports the view that our simple charge redistribution, particularly on the zinc atoms, is sufficient to find inhibitors both retrospectively and prospectively by docking.

--- PDB format file of our model ---

\begin{tabular}{|c|c|c|c|c|c|}
\hline & $A A l_{-}$ & 18 & & & \\
\hline & $\mathrm{N} 2 \mathrm{AAJ}$ & 18 & & & \\
\hline & 3 AAJ & & & & \\
\hline & 4 AAJ & & & & \\
\hline & $5 \mathrm{AAJ}$ & & & & \\
\hline & $\mathrm{UJ}$ & & & & \\
\hline & 7 AAJ & & & & \\
\hline & $8 \mathrm{AAJ}$ & & & & \\
\hline & 9 AAJ & & & & \\
\hline & AJ & & & & .230 \\
\hline & & & & & \\
\hline & $\mathrm{J}$ & 1 & & & \\
\hline & & 1 & & & \\
\hline & 6 & & & & \\
\hline & 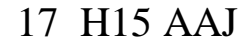 & & & & 143 \\
\hline & & & & & \\
\hline & & 1 & & & \\
\hline & & & & & \\
\hline & 21 & & & & \\
\hline & ני & & & & \\
\hline & & & & & \\
\hline & & 1 & & & \\
\hline & & & & & \\
\hline & & 1 & & & \\
\hline & & & & & \\
\hline & & & & & \\
\hline & & 1 & & & \\
\hline & 0 & & & & \\
\hline & 10 & 1 & & & \\
\hline & & & & & \\
\hline & 33 & 1 & & & .095 \\
\hline & & & & & \\
\hline & 35 & & & & \\
\hline & & & & & \\
\hline & 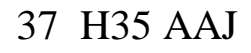 & 1 & & & \\
\hline & $38 \mathrm{C} 36$ & 1 & & & - 30.0 \\
\hline & $39 \mathrm{I}$ & & & & \\
\hline & & & & & \\
\hline & & 1 & & & \\
\hline & $421 \sqrt{2}+\mathrm{A} /$ & 10 & $48.0 / \mathrm{J}$ & $5-5.290$ & 29.01 \\
\hline
\end{tabular}




$\begin{array}{lllllll}\text { ATOM } & 43 & \text { H41 AAJ } & 18 & 48.354 & -0.920 & 31.185 \\ \text { ATOM } & 44 & \text { H42 AAJ } & 18 & 50.850 & -3.211 & 28.594 \\ \text { ATOM } & 45 & \text { H43 AAJ } & 18 & 46.863 & -2.702 & 30.073 \\ \text { ATOM } & 46 & \text { H44 AAJ } & 18 & 50.855 & -1.169 & 30.303 \\ \text { ATOM } & 47 & \text { C45 AAJ } & 18 & 45.205 & -4.288 & 31.965 \\ \text { ATOM } & 48 & \text { O46 AAJ } & 18 & 44.260 & -4.585 & 31.097 \\ \text { ATOM } & 49 & \text { O47 AAJ } & 18 & 46.397 & -4.875 & 31.901 \\ \text { ATOM } & 50 & \text { H48 AAJ } & 18 & 45.008 & -3.553 & 32.744 \\ \text { ATOM } & 51 & \text { H49 AAJ } & 18 & 46.566 & -5.251 & 30.288 \\ \text { ATOM } & 52 & \text { O50 AAJ } & 18 & 46.530 & -5.464 & 29.312 \\ \text { ATOM } & 53 & \text { ZN1 AAJ } & 18 & 44.681 & -4.760 & 28.962 \\ \text { ATOM } & 54 & \text { ZN2 AAJ } & 18 & 47.985 & -4.913 & 28.027 \\ \text { ATOM } & 55 & \text { H51 AAJ } & 18 & 44.320 & -4.173 & 24.782\end{array}$

--- end of the PDB format file

--- input to Jaguar, a quantum mechanics program from Schrodinger, Inc. --\&gen

basis=lacvp

$\operatorname{mp} 2=0$

icfit $=1$

i symm $=1$

molchg $=1$

isolv $=2$

iguess $=25$

$\&$

$\begin{array}{cccc}\text { \&zmat } & & & \\ \text { C1 } & 43.909 & -0.856 & 29.329 \\ \text { N2 } & 44.888 & -0.789 & 28.327 \\ \text { C3 } & 43.783 & -2.195 & 29.665 \\ \text { C4 } & 45.317 & -2.054 & 28.109 \\ \text { N5 } & 44.671 & -2.946 & 28.873 \\ \text { H6 } & 45.218 & 0.061 & 27.848 \\ \text { H7 } & 43.108 & -2.599 & 30.418 \\ \text { H8 } & 46.097 & -2.317 & 27.396 \\ \text { H9 } & 43.357 & -0.021 & 29.758 \\ \text { C10 } & 40.899 & -6.632 & 29.230 \\ \text { N11 } & 41.091 & -6.653 & 27.850 \\ \text { C12 } & 41.986 & -6.048 & 29.803 \\ \text { C13 } & 42.276 & -6.083 & 27.625 \\ \text { N14 } & 42.829 & -5.704 & 28.769 \\ \text { H15 } & 40.446 & -7.033 & 27.143 \\ \text { H16 } & 42.156 & -5.885 & 30.866 \\ \text { H17 } & 42.725 & -5.948 & 26.642 \\ \text { H18 } & 40.028 & -7.017 & 29.759 \\ \text { C19 } & 46.032 & -2.988 & 24.215 \\ \text { N20 } & 45.211 & -3.914 & 25.022 \\ \text { H21 } & 47.030 & -3.424 & 24.058 \\ \text { H22 } & 46.130 & -2.029 & 24.744 \\ \text { H23 } & 45.548 & -2.822 & 23.242 \\ \text { C24 } & 45.625 & -4.541 & 26.252 \\ \text { O25 } & 44.914 & -5.245 & 26.929 \\ \text { O26 } & 46.905 & -4.325 & 26.448 \\ \text { C27 } & 50.500 & -4.400 & 26.055 \\ & & & \end{array}$




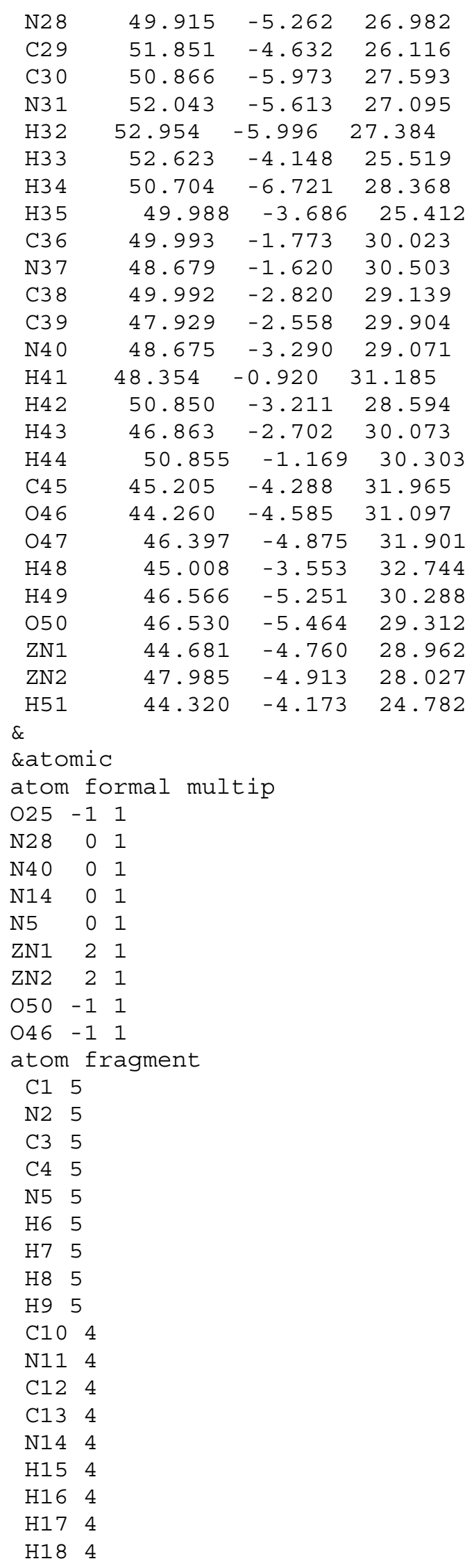




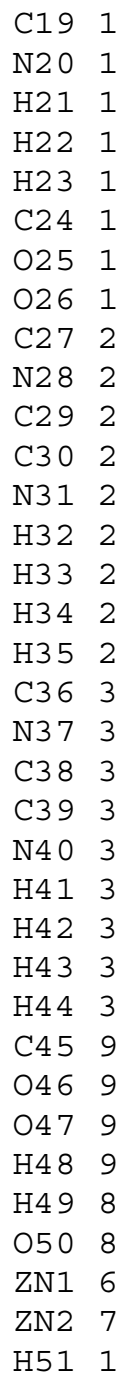

--- end of input to Jaguar, a quantum mechanics program from Schrodinger, Inc --- 
Figure S1. Molecular Weight Distribution of annotated ligand lists compared with the MDDR as a whole.

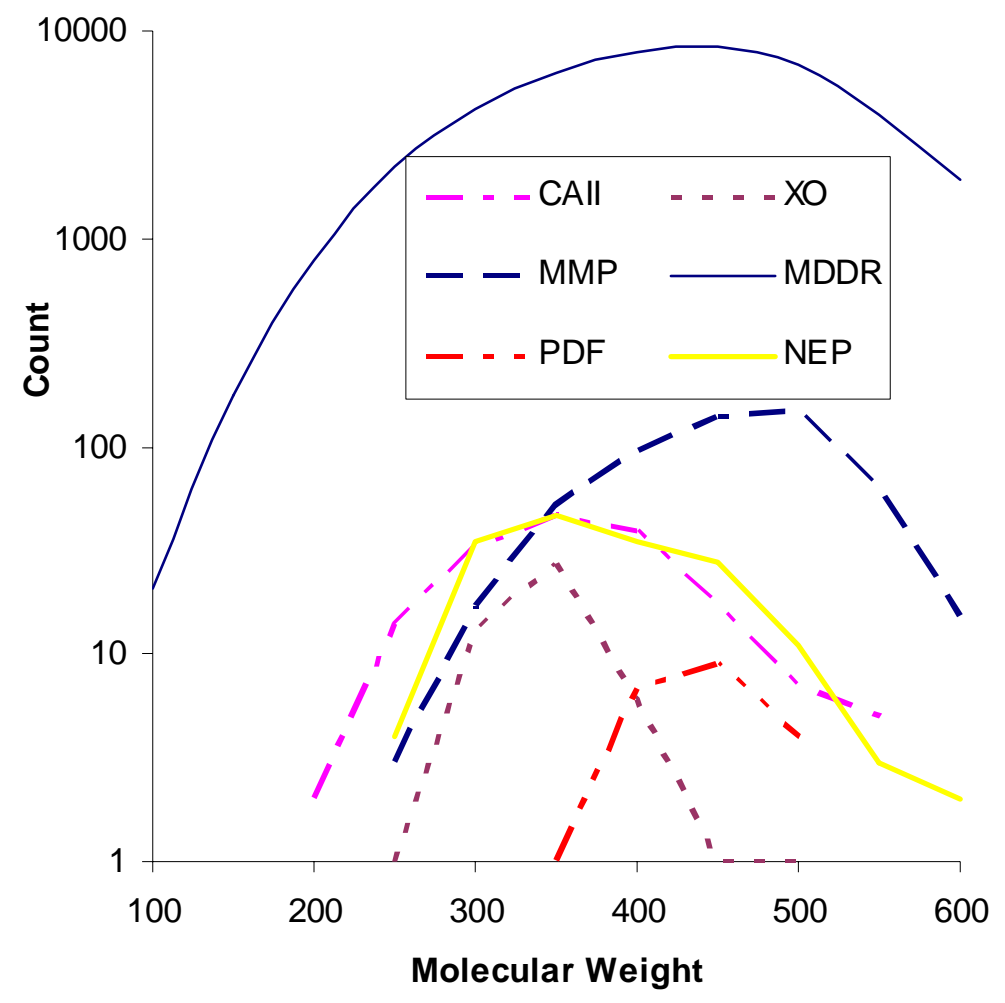

